\title{
INOX: Implantes Avaliação da resistência à torção em parafusos utilizados em implantes - um panorama brasileiro
}

\author{
The torsion strength of orthopedic implant screws - \\ a suvey of brazilian samples
}

\author{
Andrea Madeira Kliauga \\ Professora Adjunta de Engenharia de \\ Produção da Universidade Federal de \\ São Carlos - Campus de Sorocaba \\ E-mail:kliauga@ufscar.br
}

Paulo Sergio Carvalho

Pereira da Silva

CCDM - Centro de Caracterização e

Desenvolvimento de Materiais Universidade Federal de São Carlos E-mail: paulosergio@ccdm.ufscar.br

\section{Enrico J. Girodano \\ CCDM - Centro de Caracterização e Desenvolvimento de Materiais Universidade Federal de São Carlos E-mail:enrico@ccdm.ufscar.br}

\section{Ivan Della Vale Epiphanio}

CCDM - Centro de Caracterização e Desenvolvimento de Materiais Universidade Federal de São Carlos E-mail:ivan@ccdm.ufscar.br

\section{Celio Canimaga}

CCDM - Centro de Caracterização e

Desenvolvimento de Materiais Universidade Federal de São Carlos E-mail:caminaga@ccdm.ufscar.br

\section{Claudemiro Bolfarini}

CCDM - Centro de Caracterização e

Desenvolvimento de Materiais Universidade Federal de São Carlos E-mail:cbolfa@ufscar.br

\section{Resumo}

Desde 2004, o CCDM realiza ensaios mecânicos em implantes ortopédicos. Os componentes mais utilizados - e conseqüentemente ensaiados - são parafusos, comumente fabricados com aço inoxidável ASTM F138, ou liga de titânio ASTM F136. O objetivo desse trabalho é avaliar os resultados referentes aos ensaios de torção em parafusos metálicos, dando um panorama das propriedades médias dos produtos ensaiados. Tais resultados provieram de material fornecido por 15 fabricantes diferentes, num total de 451 ensaios, que cobriram quase todo o espectro de parafusos utilizados em implantes: ortodontia, parafusos pediculares, parafusos para ossos corticais e esponjosos, parafusos de bloqueio e de ancoragem. A dispersão dos resultados de ângulo de ruptura é elevada. A maioria dos resultados, no entanto, apresenta valores acima do mínimo exigido pela norma ASTM F543.

Palavras-chave: Parafusos para implantes ortopédicos, ensaios de torção.

\begin{abstract}
Since 2004, the CCDM lab performs tests on orthopedic implants. The most used (and tested) implants are screws. These are commonly made either of austenitic stainless steel ASTM F138 or of Ti-6Al-4V alloy ASTM F136. The aim of this study was to evaluate the obtained torsion test results and to present an overview of the average product properties. The results stand for 15 different producers, and 451 samples, covering almost all the screw types utilized in implants: orthodontic, anchor and tibial nail applications, and designs for pedicular, cortical and cancellous bones. The dispersion in angle of rupture was high. Nevertheless the majority of the results lied above the minimum required by the ASTM F543 standard.
\end{abstract}

Keywords: Metallic implant screws, torque measurements.

\section{Introdução}

Desde 2004, o Laboratório de Implantes do Centro de Caracterização e Desenvolvimento de Materiais (CCDM) realiza ensaios mecânicos, metalográficos, físicos, físico-químicos e químicos em implantes ortopédicos para fins de registro na ANVISA. Esses ensaios são feitos segundo normas existentes para implantes, normalmente ASTM, série F, ISO ou NBR ISO.

Não existem estatísticas sobre o uso de implantes no país, assim como faltam dados sobre falhas ocorridas após a implantação. Uma estimativa, no entanto, pode 
ser obtida pelo consumo desses insumos pelo Sistema Único de Saúde (SUS). Segundo informação divulgada pelo Datasus, para o ano de 2003, 84\% dos implantes para osteossíntese utilizados eram parafusos arruelas e pinos, seguidos de placas de fixação interna (10\%), componentes para coluna (5\%) e hastes intramedulares (1\%). Acompanhando esse perfil, 54\% dos ensaios realizados, no laboratório de implantes do CCDM, correspondem a parafusos segundo as normas ASTM F543 ou ISO 6475, seguido de ensaios de sistemas de fixação de coluna (13\%) e placas de fixação interna (10\%).

De maneira abrangente, parafusos ortopédicos podem ser classificados em parafusos corticais, quando o parafuso é fixado na região externa de maior densidade do osso (osso cortical), ou parafusos esponjosos, quando a fixação se dá, predominantemente, na região interna e de menor densidade (osso esponjoso). De acordo com T. Hirano et al. [Hirano, 1997], os parâmetros mais importantes na fixação parafuso-osso são: tamanho do parafuso, profundidade de inserção, densidade mineral óssea, técnica de inserção e torque de inserção. A influência da proporção do osso cortical, na fixação de parafusos, é mais acentuada para parafusos de menor dimensão. Por exemplo, para parafusos de ancoragem utilizados para ortodontia, a espessura do osso cortical varia entre 1,5 e 3,5mm, na mandíbula, e entre 1 e 2 mm, no maxilar [Ono, 2008]. O comprimento de mini-implantes ortopédicos é $6 \mathrm{~mm}$, portanto a proporção desse tecido irá variar de 17 a 50\% da área inserida no osso. Já os parafusos pediculares, com 4,5 a 7 mm de diâmetro e 25 a $55 \mathrm{~mm}$ de comprimento, são fixados em osso, predominantemente, esponjoso (a espessura do tecido cortical na vértebra varia de 0,2 a 2 mm [Gerlach, 1998]). Acredita-se que, aumentando a diferença diâmetro externo- alma, ou seja, a altura da rosca, aumenta-se a força de fixação ao osso, por isso parafusos esponjosos possuem altura de rosca maior do que parafusos corticais.
As normas aplicadas na avaliação dessas propriedades são a ASTM F543:2002 (Standard Specification and Test Methods for Metallic Medical Bone Screws) e a NBR ISO 6475:1997 (Implantes para cirurgia - Parafusos ósseos metálicos com rosca assimétrica e superfície inferior da cabeça de forma esférica - Requisitos mecânicos e métodos de ensaio). A NBR ISO 6475 é muito genérica e especifica apenas a determinação do torque (N.m) e do ângulo de ruptura $\left(^{\circ}\right)$. A ASTM F543 especifica a determinação do torque de escoamento em $2^{\circ}$ (N.m), torque máximo (N.m), ângulo de ruptura $\left(^{\circ}\right)$, ensaios de arrancamento (força -N e/ ou tensão de cisalhamento - $\mathrm{Pa}$ ), torque de inserção (N.m) e torque de remoção (N.m), estabelecendo parâmetros mais abrangentes de avaliação.

Existem muitos modelos de parafuso metálico e as normas não cobrem todos os tipos de projeto existentes, mas especificam alguns limites de propriedades para alguns tipos de parafusos denominados HA, HB, HC e HD. Os parafusos tipo HA ou HB têm rosca irregular e superfície arredondada na região inferior da cabeça. Os parafusos HC e HD têm rosca regular e superfície cônica na região inferior da cabeça. Para esses parafusos são estabelecidas as dimensões:

\section{Diâmetro externo \\ 2. Diâmetro da alma \\ 3. Passo da rosca \\ 4. Perfil da rosca \\ 5. Altura da rosca}

O ensaio de torção tem como finalidade avaliar as propriedades mecânicas do projeto do parafuso. Na Norma ASTM F 543, são dados alguns limites mínimos de propriedade para parafusos HA (tipicamente de aplicação em osso cortical) e HB (tipicamente de aplicação em osso esponjoso) no ensaio de torção, independente do tipo de material de fabricação (Tabela 1).

Para outros projetos de rosca, não existe nenhuma publicação que delimite as propriedades mínimas desejáveis. No entanto, os valores da Tabela 1 po- dem servir de guia, esperando-se que variações de projeto não diminuam a resistência com relação à esses projetos tradicionais.

Com o intuito de verificar se parafusos HA e HB apresentem níveis seguros de resistência, durante a sua inserção, foi feita uma revisão dos valores de torque de inserção ou de remoção de parafusos metálicos (titânio ou aço inox) em ossos medidos em cadáveres ou em vivo (ferramenta cirúrgica instrumentada) [Bühler, 1998, Buijs, 2003, Buijis, 2007, Gerlach, 1998, Kasuisha, 2005, Kowow, 1996, Lehrman, 2008, Lei, 2006, Mayers, 1996, Morais, 2007, Ono, 2008, Yerby, 2001,Yilerim, 2006, Zink, 1996]. A comparação com os torques de ruptura mostra (ver figura 1 ) que parafusos metálicos com desenho do tipo HA apresentam valores de torque seguros para a maioria das aplicações cirúrgicas, mas, para as dimensões de parafusos ortodônticos (1,5-2mm de diâmetro externo), os torques de inserção podem ser críticos. Para parafusos HB 4,0, onde há maior diferença entre raio externo e alma, pode ocorrer fratura do parafuso durante o procedimento cirúrgico.

O objetivo desse trabalho é avaliar os resultados referentes aos ensaios de torção em parafusos metálicos dando um panorama das propriedades médias dos produtos ensaiados. Tais resultados provieram de material fornecido por 15 fabricantes diferentes, num total de 451 ensaios, que cobriram quase todo o espectro de parafusos utilizados em implantes: ortodontia, parafusos pediculares, parafusos para ossos corticais e esponjosos, parafusos de bloqueio e de ancoragem.

\section{Materiais e métodos}

O CCDM realiza ensaios para determinação das propriedades de torção, nos quais se obtêm os valores de torque de escoamento (TE; N.m), torque de ruptura (LT; N.m) e ângulo de ruptura $\left(\mathrm{AR} ;^{\circ}\right)$. Foi utilizada uma máquina de ensaios Termo Mec Orto, desenvolvida no Departamento de Engenharia de Materiais da UFSCar, equipada com 
Tabela 1 - Limites mínimos de torque na ruptura (LT) e ângulo de ruptura (AR) para parafusos HA e HB, segundo a norma ASTM F543.

\begin{tabular}{l|c|c|c}
\hline \multicolumn{2}{c|}{ Tipo e tamanho } & $\begin{array}{c}\text { Valores mínimos } \\
\text { aceitáveis }\end{array}$ \\
\hline & $\begin{array}{c}\text { Øalma } \\
(\mathrm{mm})\end{array}$ & $\begin{array}{c}\mathrm{TM} \\
(\mathrm{N} . \mathrm{m})\end{array}$ & $\begin{array}{c}\text { AR } \\
(\mathrm{o})\end{array}$ \\
\hline HA1,5 & 1,1 & 0,20 & 150 \\
$\mathrm{HA2}, 0$ & 1,3 & 0,35 & 150 \\
$\mathrm{HA} 2,7$ & 1,9 & 1,00 & 180 \\
HA3,5 & 2,4 & 2,30 & 180 \\
HA4,0 & 2,9 & 4,00 & 180 \\
HA4,5 & 3,0 & 4,40 & 180 \\
HA5,0 & 3,5 & 5,50 & 180 \\
HB4,0 & 1,9 & 1,30 & 90 \\
HB6,5 & 3,0 & 6,20 & 90 \\
\hline
\end{tabular}

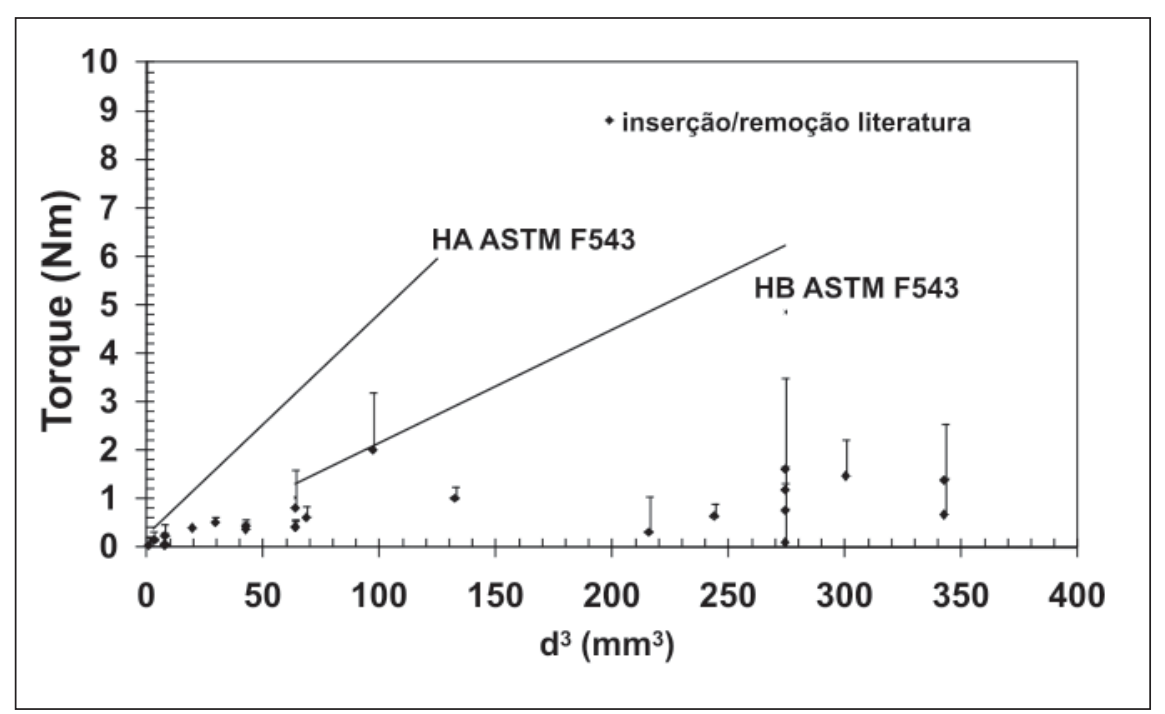

Figura 1 - Comparação com torques de ruptura previstos para parafusos HA e HB e torques de inserção ou remoção em osso encontrados na literatura [Bühler, 1998, Buijs, 2003, Gerlach, 1998, Kasuisha, 2005, Kowow, 1996, Lehrman, 2008, Lei, 2006, Mayers, 1996, Morais, 2007, Ono, 2008, Yerby, 2001,Yilerim, 2006, Zink, 1996].

um motor cervo-elétrico de 6 KVA com velocidade variável de 1 a $2.000 \mathrm{rpm}$ e célula de carga com capacidade de 20 N.m. A deformação e a taxa de deformação são calculadas a partir das medidas do ângulo de rotação realizadas por um transdutor de rotação. A reprodutibilidade e a repetitividade do equipamento foram testadas em ensaios de torção de corpos-de-prova cilíndricos de aço inoxidável ASTM F136 e levantamento de curvas tensão e de deformação equivalentes. A aquisição de dados é realizada por um computador interligado à máquina, que, com um programa, controla os ensaios. Os ensaios foram realizados com velocidade de $2 \mathrm{rpm}$. A Figura 2 mostra um arranjo típico de ensaio de torção.

No período janeiro 2007 - maio 2008, o CCDM realizou 451 ensaios de torção, cada ensaio representando uma média dos resultados obtidos em pelo menos 5 amostras. Os parafusos ensaiados atendem a várias finalidades: ortodontia, parafusos pediculares, parafusos para ossos corticais e esponjosos, parafusos de bloqueio e de ancoragem. Durante a contratação do ensaio, nem sempre é fornecido o diâmetro da alma, por isto todos os resultados foram comparados com relação ao diâmetro externo. A Tabela 2 apresenta a quantidade de ensaios realizados, classificando os parafusos por seu diâmetro, pela sua aplicação (osso esponjoso ou cortical segundo indicação do fabricante) e pelo número de empresas representadas em cada amostragem.

Os parafusos ensaiados foram fabricados com aço inoxidável ASTM F138 ou liga de titânio ASTM F136. $\mathrm{Na}$ análise dos resultados, não foi feita nenhuma distinção quanto ao tipo de material de fabricação.

\section{Resultados e discussão}

A partir desse ponto, as denominações HA e HB referem-se a parafusos com projeto, respectivamente, para ossos corticais e esponjosos.

No presente trabalho, foram analisados os resultados das propriedades de torção, através do torque máximo e do ângulo de ruptura, determinados de acordo com as especificações da ASTM F 543-02, Anexo A1. No entanto foram considerados os resultados do torque e do ângulo de ruptura (TR), ao invés do torque máximo (TM). A Figura 3 mostra as curvas torque-ângulo de uma série de ensaios com a ilustração dos parafusos ensaiados após a ruptura, mostrando que esses valores são coincidentes.

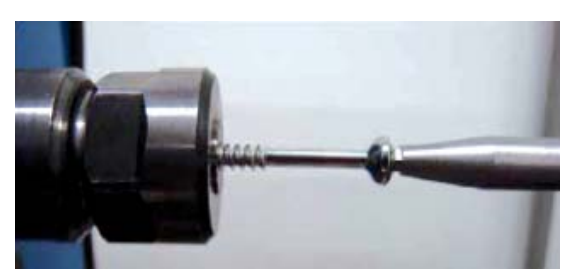

Figura 2 - Arranjo experimental aplicável à maioria dos parafusos ensaiados. 
No ensaio padronizado, o comprimento útil ao qual é aplicado o torque é de 5 filetes para parafusos com rosca total ou parcial. Se o parafuso for pequeno ou o comprimento de rosca insuficiente e não permitir a fixação, 20\% da região da rosca é sujeita ao ensaio. Nessa configuração, o parafuso pode, ignorando-se o fator de concentração de tensão $\mathrm{K}$, associado ao projeto de cada rosca, ser modelado como uma barra engastada e sujeita à torção na extremidade livre, sendo que a secção solicitada é a alma do parafuso. A tensão de cisalhamento máxima atuante em torção, t, será dada pela expressão:

$\tau=16 \mathrm{~T} / \pi \mathrm{d}^{3}$

onde T é o torque e d o diâmetro da alma.

No entanto, a informação sobre o diâmetro da alma nem sempre consta nas publicações técnicas da área, sendo mais comum a caracterização do parafuso pelo diâmetro externo da rosca, pois esse parâmetro está diretamente relacionado com os torques de inserção e de remoção e com a tendência ao arrancamento durante e após a implantação. São, também, mais freqüentes os estudos sobre a qualidade da fixação em ossso e sobre as tensões de inserção/remoção do que sobre as propriedades mecânicas em torção.

O gráfico da Figura 4 mostra uma comparação dos valores mínimos para torque máximo estabelecidos pela ASTM F 543 e de dados obtidos na literatura [Glauser, 2003, Hirano, 1997]. Os resultados encontrados situam-se entre os valores especificados para parafusos HA e HB, independente do material (titânio ou aço inox) de fabricação utilizado.

A Figura 5 mostra os resultados dos ensaios realizados comparados aos valores aceitáveis para parafusos HA e HB. Observa-se que, para parafusos de até 4,5 mm de diâmetro externo, não ocorre muita dispersão de valores de torque, sendo que a média das medidas coincide com a prevista para parafusos do tipo HA. A maioria dos valores de torque encontra-se acima do mínimo aceito para parafusos HB.

Tabela 2 - Parafusos ensaiados.

\begin{tabular}{c|c|c|c}
\hline $\begin{array}{c}\text { Diâmetro } \\
\text { externo } \\
(\mathbf{m m})\end{array}$ & $\begin{array}{c}\text { Número } \\
\text { de ensaios }\end{array}$ & Aplicação & $\begin{array}{c}\text { Número de } \\
\text { Empresas }\end{array}$ \\
\hline 1,5 & 38 & $38 \mathrm{C}$ & 4 \\
\hline 2,0 & 40 & $40 \mathrm{C}$ & 3 \\
\hline 2,7 & 35 & $35 \mathrm{C}$ & 5 \\
\hline 3,5 & 152 & $152 \mathrm{C}$ & 6 \\
\hline 4,0 & 51 & $51 \mathrm{E}$ & 7 \\
\hline 4,5 & 61 & $20 \mathrm{C} ; 41 \mathrm{E}$ & 5 \\
\hline 5,0 & 15 & $15 \mathrm{C}$ & 4 \\
\hline 6,0 & 15 & $5 \mathrm{C} ; 10 \mathrm{E}$ & 3 \\
\hline 6,5 & 36 & $18 \mathrm{C} ; 18 \mathrm{E}$ & 7 \\
\hline 7,0 & 8 & $8 \mathrm{E}$ & 2 \\
\hline
\end{tabular}

$\mathrm{C}=$ crtical; $\mathrm{E}=$ esponjoso $\mathrm{n}=$ empresa nacional $; \mathrm{i}=$ empresa importadora

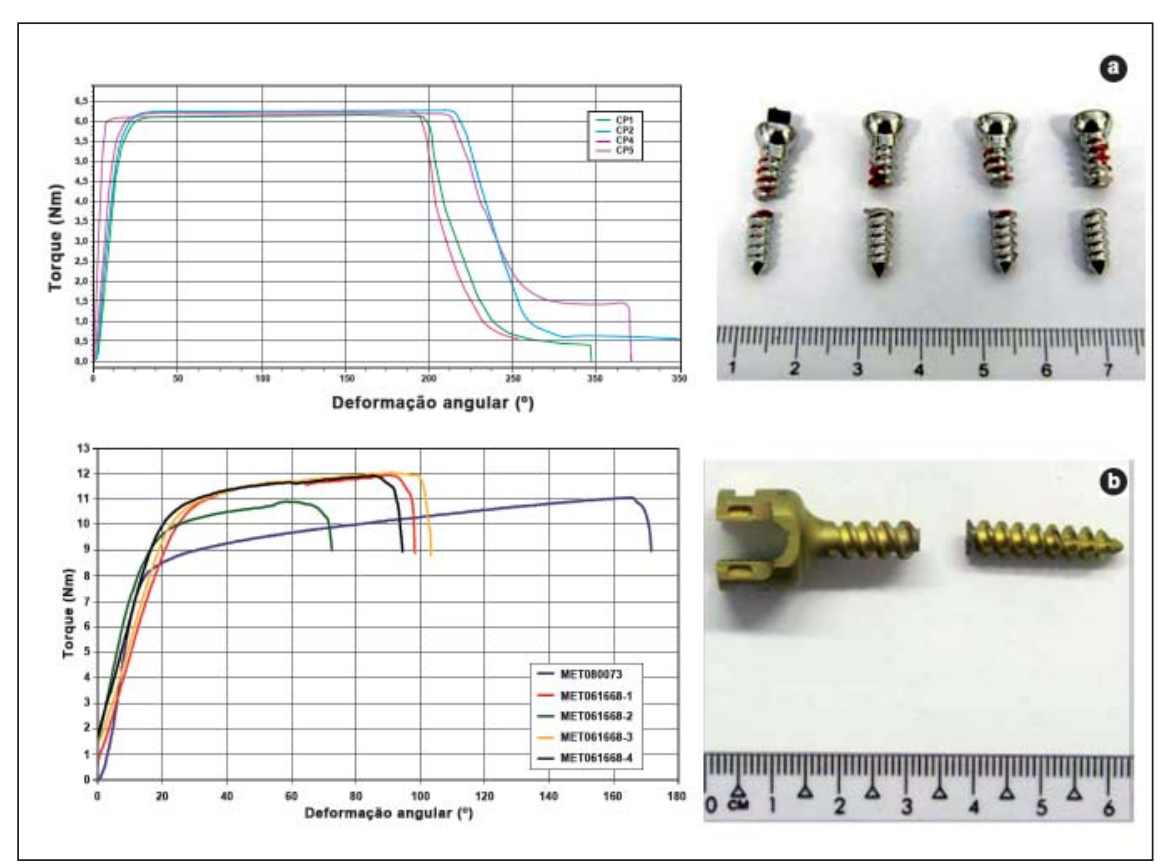

Figura 3 - Exemplos de curvas torque-ângulo obtidas para dois tipos de parafusos a) cortical $\varnothing 3 \mathrm{~mm} ;$ b) pedicular $\varnothing 6 \mathrm{~mm}$.

O ângulo de ruptura não mostrou qualquer tipo de correlação com o diâmetro do parafuso (Figura 6), mas a maioria dos produtos (70\%) tem valores acima do mínimo esperado para parafusos HA e HB.

Foram selecionados 5 fabricantes, com maior número de amostras de até 4,5 mm de diâmetro, apresentados na
Figura 7. Não ocorre diferença significativa entre fabricantes, todos eles acompanhando os limites previstos para parafusos de implantes.

\section{Conclusões}

A maioria dos parafusos atinge níveis de ruptura mínimos previstos 


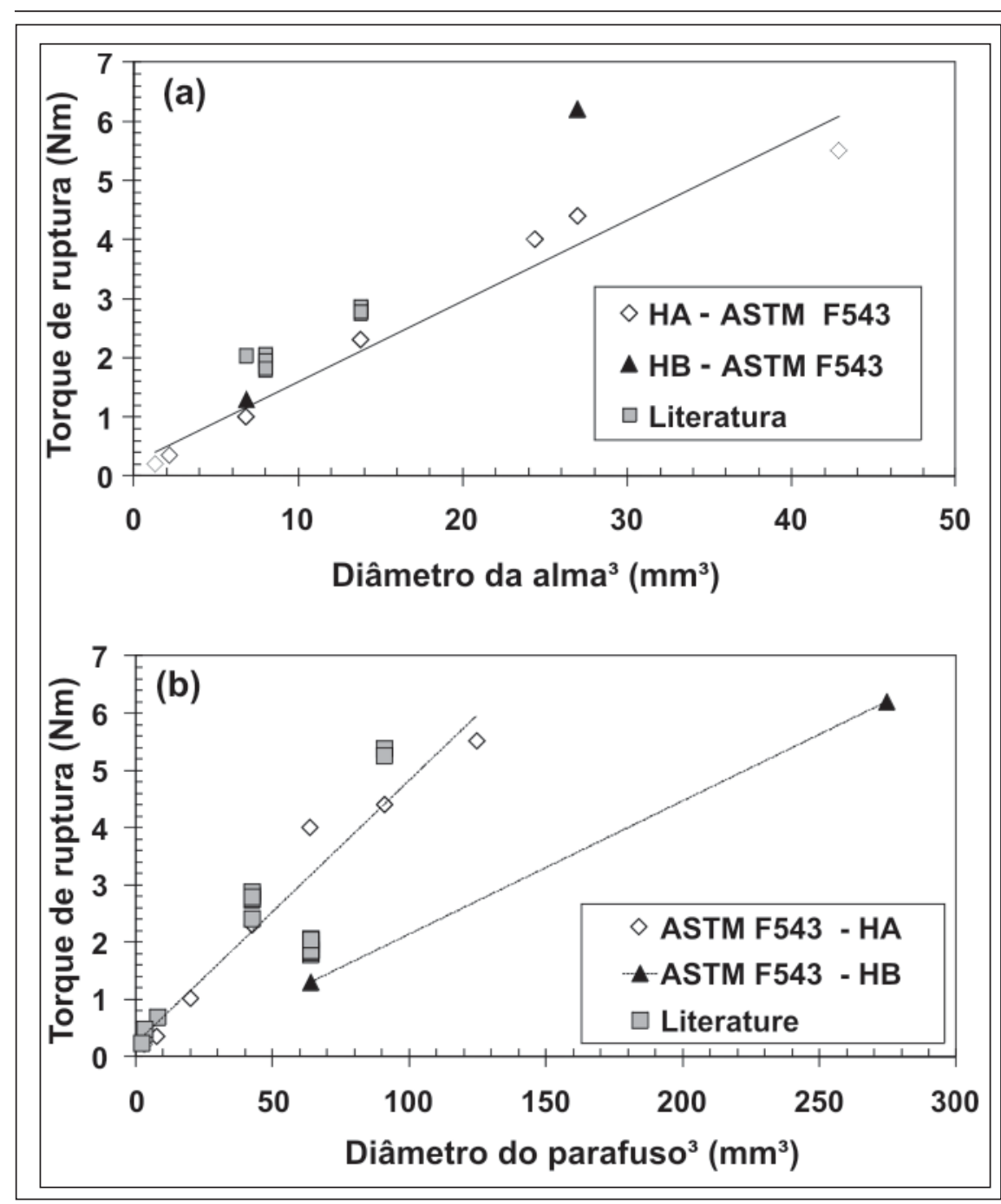

Figura 4 - Relação entre torque de ruptura e a) diâmetro da alma; b) diâmetro externo da rosca do parafuso.

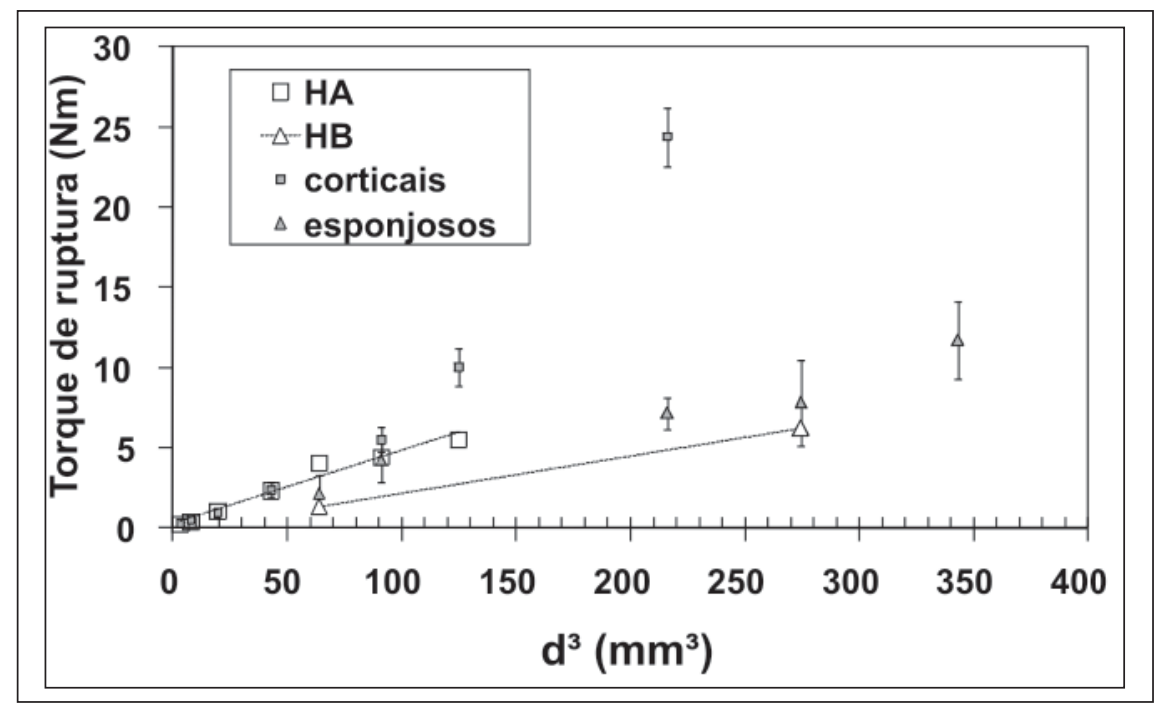

Figura 5 - Relação torque de ruptura - diâmetro externo dos parafusos ensaiados em comparação com os limites mínimos exigidos pela norma ASTM F543 para parafusos HA e HB. pela norma ASTM F543. O dimensionamento é adequado para o nível de tensão encontrado durante a maioria dos procedimentos cirúrgicos. A dispersão dos resultados do ângulo de ruptura é elevada. A maioria dos resultados, no entanto, apresenta valores acima do mínimo exigido pela norma ASTM F 543.

\section{Referências bibliográficas}

ASTM F543:2002 - Standard specification and test methods for metallic medical bone screws.

BÜHLER D. W. et al. Moments and forces during pedicle screw insertion: in vitro and in vivo measurements. Spine, v. 23, p. 1220-1227, 1998.

BUIJIS G. J. et al. Torsion strength of biodegradable and titanium screws: a comparison. J. Oral Maxillofac. Surg., p. 2142-2147. 2007.

BUIJS G. J. et al. Torsion strength of biodegradable and titanium screws: a comparison. J. Oral Maxillofac Surg, p.2142-2147, 2007.

CHRISTIENSEN, F. B. et al. Titaniumalloy enhances bone-pedicle screw fixation: mechanical and histomorphometrical results of titanium-alloy versus stainless steel. Euro Spine J. v. 9, p. 97-103, 2000.

GLAUSER C. R. et al. Mechanical testing of small fracture implants ofr comparison of insertion and failure torques, Arch. Orthop Trauma Surg., v.123, p. 388-391, 2003.

GERLACH, K.L., GROBEL, K.H., HEIDMANN, W., KOLLNER, H.G. Influence of different pilot hole sizes on torque measurements and pullout analysis of oteosynthesis screws. J. Craniomaxillofac. Surg., v. 26, p. 50-55, 1998.

HIRANO, T. et al. Structural characteristics of the pedicle and its role in screw stability. Spine, v.21, p. 2504-2510, 1997.

KASUISHA, T. O. et al. Insertion torque of lumbar screw during surgery. $\boldsymbol{J}$. Orthop. Sci, v.10, p. 133-136, 2005.

KWOW, A. W. L. et al. Insertion torque and pull-out strengths of conical and cylindrical pedicle screws in cadaveric bone. Spine, v. 21, p. 24292434, 1996. 


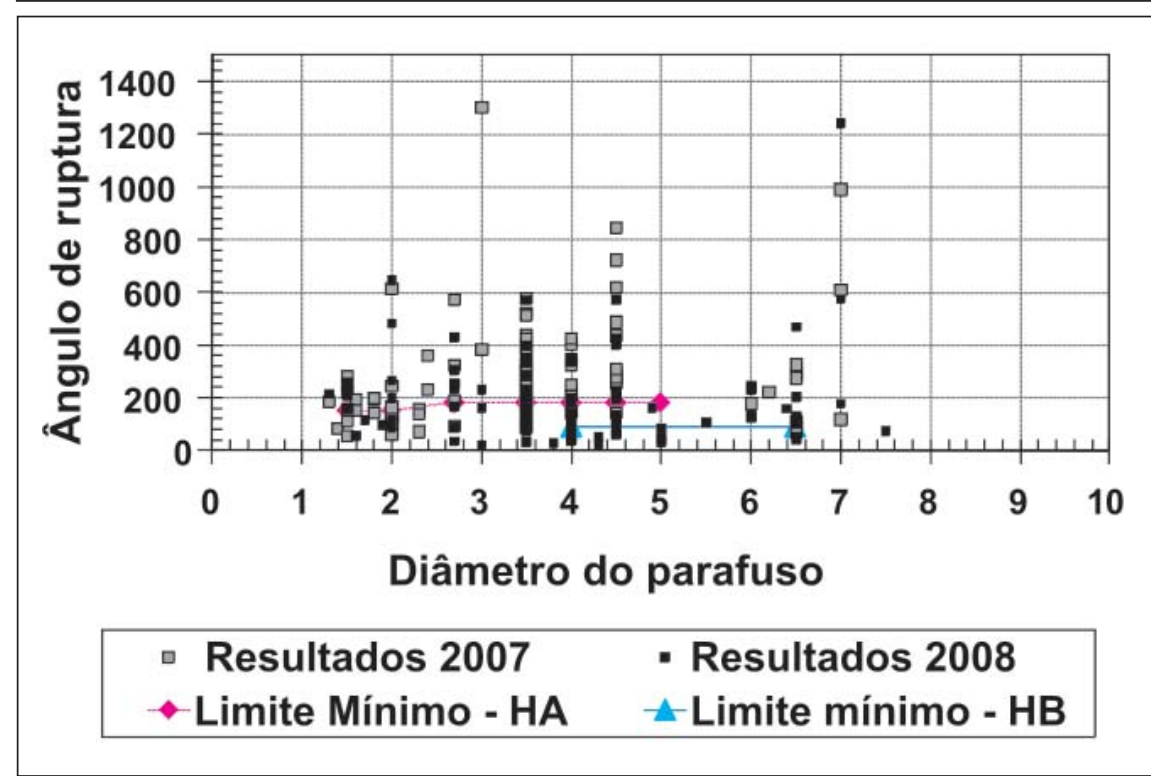

Figura 6 - Ângulo de ruptura em função do diâmetro dos parafusos ensaiados.

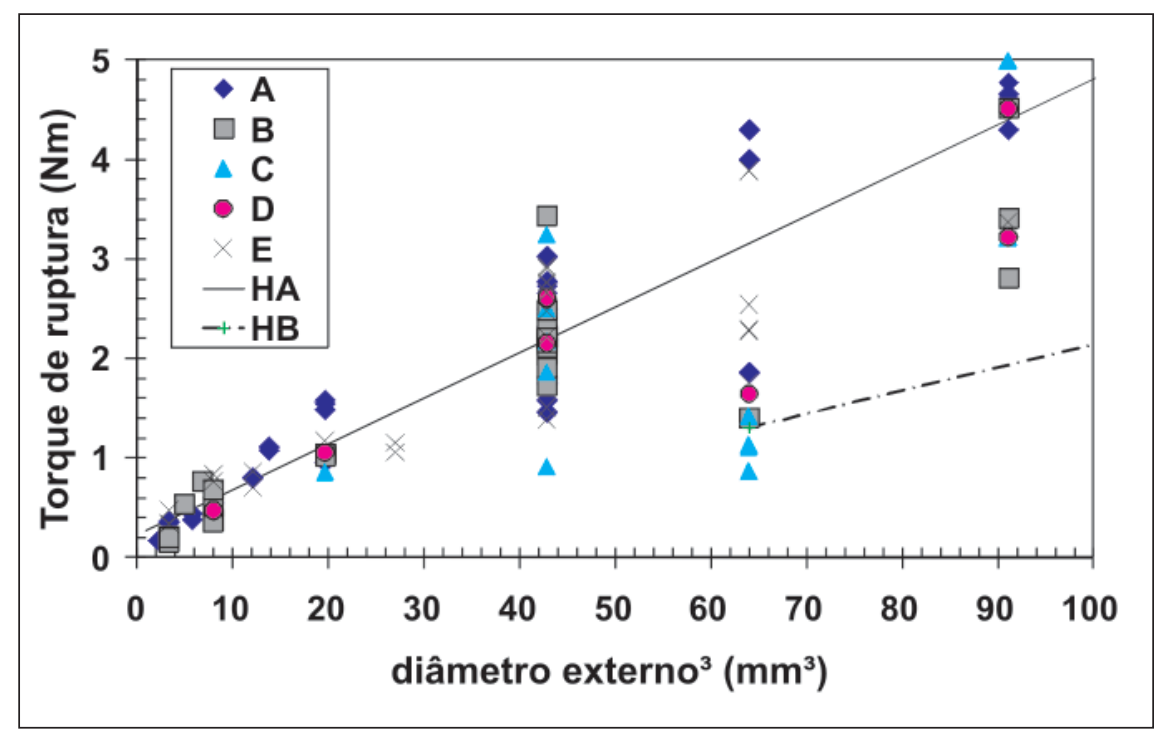

Figura 7 - Resultados de torque de ruptura ( $\mathrm{N}$ m) em função do diâmetro externo do parafuso $\left(\mathrm{mm}^{3}\right)$. Fabricantes nacionais: A, C, D e E. Importadora: B.
LEHRMAN, R. A. et al. Salvage of C2 pedicle and pars screws using the intralaminar technique. Spine, v.33, p. 960-965, 2008.

LEI, W., WU, Z. Biomechanical evaluation of expansive pedicle screw in calf vertebrae. Euro Spine J., v.15, p. 321-326, 2006.

MAYERS, B. et al. The role of imaging and in situ biomedical testing in assessing pedicle screw pull-out strength. Spine, v.21, p. 1962-1968, 1996.

MORAIS, L. S. et al. Titanium alloy miniimplants for orthodontic anchorage: immediate loading and metal iron release. Acta Biomaterialia, v. 3, p. 331-339, 2007.

NBR ISO 6475:1997 Implantes para cirurgia - parafusos ósseos metálicos com rosca assimétrica e superfície inferior da cabeça de forma esférica - requisitos mecânicos e métodos de ensaio.

ONO, A., MOTOY OSHI, M., SHIMIZU N., Cortical bone thickness in the buccal posterior region for orthodontic miniimplants. Int. J. Oral Maxillofac. Surg., v. 37, p. 334-340, 2008.

YERBY, S. et al. Effect of cutting flute design on cortical bone screw insertion torque and pullout strength. J. Orthop. Trauma, v.15, p. 216-221, 2001.

YILDERIM, O. Y. et al. Hydroxyapatite dip coated and uncoated titanium polyaxial pedicle screws: an in vivo bovine model. Spine, v.31, p. 215-220, 2006.

ZINK, P.M. Performance of vertebral spondylodesis screws in cervical vertebrae of varying bone mineral density, Spine, v.21, p. 45-52, 1996.

Artigo recebido em 09/08/2009 e aprovado em 18/01/2010.

\section{A REM tem novo endereço: FUNDAÇÃO GORCEIX - REM Rua Carlos Walter Marinho Campos, 57 Bairro: Vila Itacolomy 35400-000 - Ouro Preto - MG $\begin{array}{ll}\text { (31) } 3551-4730 & \text { (31) 3559-7408 }\end{array}$}

